\title{
Monotonous MHC sing in troubled times
}

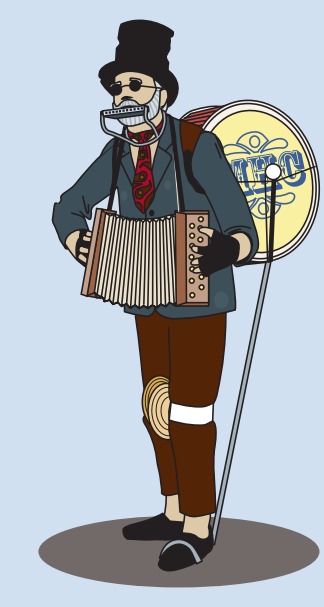

Mouse Qa- $1^{\mathrm{b}}$ and its human homologue, HLA-E, are highly conserved MHC class I-like molecules that protect host cells from cytotoxic lymphocytes by presenting a single self peptide, Qa-1 determinant modifier (Qdm) from the signal sequence of classical MHC class I molecules, to inhibitory natural killer (NK)-type receptors. Now, a study by Oliveira et al. shows that in cells with defects in the MHC class I antigen processing pathway, Qa-1 ${ }^{\mathrm{b}}$-bound Qdm is replaced with a diverse range of self peptides and this leads to elimination of these cells by $\mathrm{CD} 8^{+}$cytotoxic T lymphocytes (CTLs).

Following immunization of mice with transporter associated with antigen processing (TAP)-deficient syngeneic cells, the authors isolated Qa-1 $1^{\mathrm{b}}$-restricted $\mathrm{CD} 8^{+} \mathrm{T}$ cell clones that showed cytotoxic activity against TAP-deficient cell lines. The isolated $\mathrm{T}$ cell clones had a classic CTL phenotype and a diverse $\mathrm{T}$ cell receptor (TCR) repertoire. TAPdeficient cells could not present Qdm but, instead, presented new peptides on Qa-1 ${ }^{\text {b }}$. Experiments with specific blocking antibodies ruled out the use of NK-type receptors by the $\mathrm{T}$ cell clones and showed their cytotoxic activity was mediated by TCR recognition of the Qa- $1^{\mathrm{b}}$ presented peptides. Cytotoxicity of the $\mathrm{CD}^{+} \mathrm{T}$ cells could be inhibited by exogenous loading of TAPdeficient cells with excess Qdm peptide, suggesting that in healthy cells the high-affinity binding of Qdm to Qa- ${ }^{\mathrm{b}}$ prevents presentation of other self peptides. Interestingly, the Qa-1 ${ }^{\mathrm{b}}$-restricted $\mathrm{CD} 8^{+} \mathrm{T}$ cell clones showed CTL activity against several tumour cell lines with known antigen-processing defects.

Using biochemical purification and tandem mass spectrometry, the authors next assessed the nature of the non-Qdm peptides presented by Qa- $1^{\mathrm{b}}$ molecules. These peptides were derived from endogenous proteins and were extremely diverse. Screening of the alternative Qa-1 ${ }^{\mathrm{b}}$-presented self peptides identified several immunogenic peptides, which had no common amino acid motif and were derived from different housekeeping proteins - further supporting evidence of the diversity of the alternative Qa- $1^{\text {b }}$ self-peptide repertoire.

Although this study focused on mouse Qa-1 ${ }^{\mathrm{b}}$, the authors speculate that owing to the conserved structure and function of these MHC class I-like molecules, HLA-E might have a similar role in promoting human CTL activity against transformed or virus-infected tissue cells.

Yvonne Bordon

ORIGINAL RESEARCH PAPER Oliveira, C. C. et al. The nonpolymorphic MHC Qa- $1^{b}$ mediates CD8 ${ }^{+} T$ cell surveillance of antigen-processing defects. J. Exp. Med. 28 Dec 2009 (doi:10.1084/ jem.20091429) 